\title{
Temporal interval production and processing in working memory
}

\author{
CLAUDETTE FORTIN and RICHARD BRETON \\ Université Laval, Quebec, Canada
}

\begin{abstract}
Short-term memory or working memory has been proposed as a cognitive structure contributing to time estimation. Thus, in a previous experiment, retrieving a stored item during a temporalinterval production lengthened the interval in proportion to the number of items in the memory set. In the present study, this issue was analyzed further by testing whether the proportional lengthening is induced by the load itself (i.e., the number of items) or by comparing the probe with memorized items. In a first experiment, a memory set was maintained during a temporal production, and the comparison of the probe with memorized items was postponed until the end of time production. Varying the number of items in the memory set had no effect on temporal intervals produced during its retention, suggesting that mental comparison was the source of the lengthening of time intervals. In succeeding experiments, tasks requiring processing in working memory but involving no memory load were combined with temporal production. In Experiment 2, increasing the number of syllables in a rhyme-judgment task proportionally lengthened temporal intervals that were produced simultaneously. In Experiment 3, increasing the amount of mental rotation in a task involving visuospatial processing also lengthened simultaneous temporal production. This interference between processing in working memory and time estimation suggests that working memory, defined as a work space for active processing of current information, contributes to time estimation.
\end{abstract}

Time estimation is often represented as relying on the activity of two main components of a timing system: a pacemaker or a clock generating pulses, and a counter accumulating them and signaling when the number of pulses reaches a criterion value corresponding to a given duration (e.g., Fetterman \& Killeen, 1990; Gibbon, Church, \& Meck, 1984; Hicks, Miller, \& Kinsbourne, 1976; Rousseau, Picard, \& Pitre, 1984). In prospective time estimation, simultaneous nontemporal processing may result in a shortening of the estimated interval (Hicks, Miller, Gaes, \& Bierman, 1977); in a temporalinterval-production paradigm, nontemporal processing lengthens the produced time interval (Fortin \& Rousseau, 1987). These data suggest that some nontemporal activities may interrupt pulse accumulation; in fact, such data have been used to assume attentional control in time estimation, thus forming the category of attentional models of time estimation (Block, 1990). However, using a concurrent processing methodology in which they interpolated nontemporal processing in a temporal interval produced by a subject, Fortin, Rousseau, Bourque, and Kirouac (1993) showed that attention-demanding search tasks did not interfere with time estimation if these

This research was supported by Grant OGP0046422 from the Natural Science and Engineering Research Council of Canada to the first author. The second experiment of this study was run as part of a master's thesis by the second author. Correspondence should be addressed to Claudette Fortin, École de psychologie, Université Laval, Quebec, PQ, Canada GIK 7P4 (e-mail: fortincl@vm1.ulaval.ca). search tasks did not require processing in short-term memory.

In Fortin et al.'s (1993) study, four memory or visual search tasks were successively combined with time production. In control conditions, these four search tasks were shown to lead to comparable functions of reaction time versus set size. The criterion of increasing reaction time as a function of set size is usually accepted as an index of attentional resource demands (e.g., Schneider \& Shiffrin, 1977; Treisman \& Gelade, 1980). Although the reaction time functions suggested that the search tasks were of comparable difficulty levels, they were shown not to produce similar interference patterns with time production. Actually, the amount of interference seemed to be determined by the processing demands in short-term memory. Thus, in Experiment 1, 1 to 6 items were memorized, these items having to be compared to a single visual target. That produced a proportional increase of time intervals with set size. In Experiment 2, the memory demands in the search task were reduced, since the memory-set size was always one. One to five visually presented targets had to be compared with the memorized item. This task still interfered with time production, but to a lesser extent than did the first one. In Experiments 3 and 4, a single item, always the same throughout the experiment, was to be detected in a visual display including 1 to 15 items. In these two experiments, although the difficulty level was comparable to that of the two preceeding experiments, memory demands were practically null, and so was the interference with time production. 
These results integrate time estimation research and theorizing in attention research, where interference specificity is taken as evidence for sharing one of multiple pools of capacity (e.g., Wickens, 1984). However, to conclude that interference occurs between memory search and time estimation leaves open the question of which components of the memory search interfere with time estimation. Thus, the proportional lengthening of temporal intervals could have been related to the memory load or to the successive comparing of the target with these items.

An experimental trial is illustrated in Figure 1A. First a warning signal was presented; it disappeared when the subject pressed a key to begin the trial. This keypress triggered the successive presentation of memoryset digits $(n=1-6)$. A fixation point was then displayed; when ready, the subject began the interval production by pressing a key. Five hundred milliseconds after this keypress, the fixation point disappeared and was replaced by the probe, which remained present until the subject ended the time interval production by pressing one or the other of two keys-the left one if the probe was negative (not a member of the memory set), the right one if the probe was positive. This was followed by feedback ("correct" or "error") about the memory search. It is important to note that, with a single keypress, the subject provided the response to the memory search (by choosing the location of the key) and ended the temporal interval (by deciding the moment at which the key was pressed). Thus, even if the comparison of the probe with the memorized items had to be performed during the time interval estimation, it was not speeded and could even be postponed almost until the end of the interval. Therefore, two components of the nontemporal processing task could have induced the proportional lengthening of the temporal interval: the memory load, or the comparing of the target with members of the memory set.

In Experiment 1, the experimental conditions were identical to those shown in Figure 1A, with the exception that the probe was presented immediately after, instead of during, the temporal interval. As illustrated in Figure 1B, this implies that the memory set must be maintained in memory during most of the interval; after the end of the interval production, the subject could compare the target with memory-set items. This task bears some similarity to the concurrent-memory-load technique, in which a task is performed during the retention interval of a concurrent short-term-memory task (see, e.g., Logan, 1979); the effect of varying the load in memory is considered to be an index of attention demands in the concurrent task.

Comparing trial sequences in Figures $1 \mathrm{~A}$ and $1 \mathrm{~B}$ allows one to see that probe presentation at the end of the temporal production results in dissociating some components of the memory search task. In the sequence illustrated in Figure 1A, comparison of the probe with the members of the memory set had to be performed during the time interval. In the sequence shown in Figure 1B, comparison had to be delayed until the end of the interval. During the interval, the memory-set items had to be maintained in memory. Consequently, an interference proportional to the memory load, between memory search and time production, could be explained by the load, but not by a comparison of the probe with the memorized items.

\section{EXPERIMENT 1}

\section{Method}

Subjects. Seven subjects, 2 male and 5 female, between 21 and 31 years old (mean age, $25 ; S D=2.4$ ), took part in this experiment. They were all undergraduate or graduate students at Universite Laval. They were paid $\$ 5$ per session for their participation. In all the experiments of the present study, the subjects were naive regarding the hypothesis of the experiment. None of the subjects in Experiment 1 or in any of the succeeding experiments participated in another experiment of the present study.

Stimuli and Apparatus. Stimulus and feedback presentations were controlled by a PC-compatible computer running MEL (Micro Experimental Laboratory) software. The subject was seated in front of a color screen (VGA, Amdek Color 600), which was placed about $60 \mathrm{~cm}$ from his/her eyes. One hand rested on a response box (rb-100/n) that could be moved to place it in a position comfortable for the subject. Three adjacent keys of the response box were used in this experiment. The middle key was used to produce the temporal interval. The left and right keys were used to give the response in the memory search task: pressing the left key indicated a negative (target-absent) trial; the right key, a positive (target-present) trial. The experiment was run in a soundattenuated test chamber.

The set of items used in the experiment comprised 10 digits $(0$, $1, \ldots, 9)$. The memory-set size ( $n=1-6$ different digits) and the probe digit $(n=1)$ were selected randomly and varied from trial to trial. The digits, white on a black background, subtended $0.2^{\circ}$ of visual angle in height and $0.4^{\circ}$ in width.

Procedure. Subjects were run individually in five sessions: three temporal production practice sessions, and two experimental sessions in which the memory search task was introduced to be combined with the temporal production task. Practice as well as experimental sessions included five blocks, with a 30 -sec rest between blocks. Sessions lasted between 30 and $45 \mathrm{~min}$.

At the beginning of the first practice session, examples of the target interval were presented to the subject without referring to conventional units of time; the experimenter, using a chronometer, pressed a key two times in succession, with a 2 -sec interval between the two keypresses. The subject was then asked to produce the target interval, correcting it with the feedback that was provided in the practice sessions: after each temporal interval produced by the subject, visual feedback was presented on the screen, informing the subject that the temporal production was too long, too short, or correct, within a $200-\mathrm{msec}$ window centered on the 2-sec standard.

After these preliminary trials, the first temporal production practice session began. In this session, as in the two following practice sessions, a trial started with a visual warning signal indicating to the subject that a new trial was beginning. When ready, the subject started the interval production by pressing the middle key. After the subjective interval had elapsed, the subject ended the interval by pressing the same key anew. The target interval was the same throughout the experiment-that is, 2 sec.

In the three temporal production practice sessions, there were five 48-trial blocks. In the first four blocks, feedback about the 

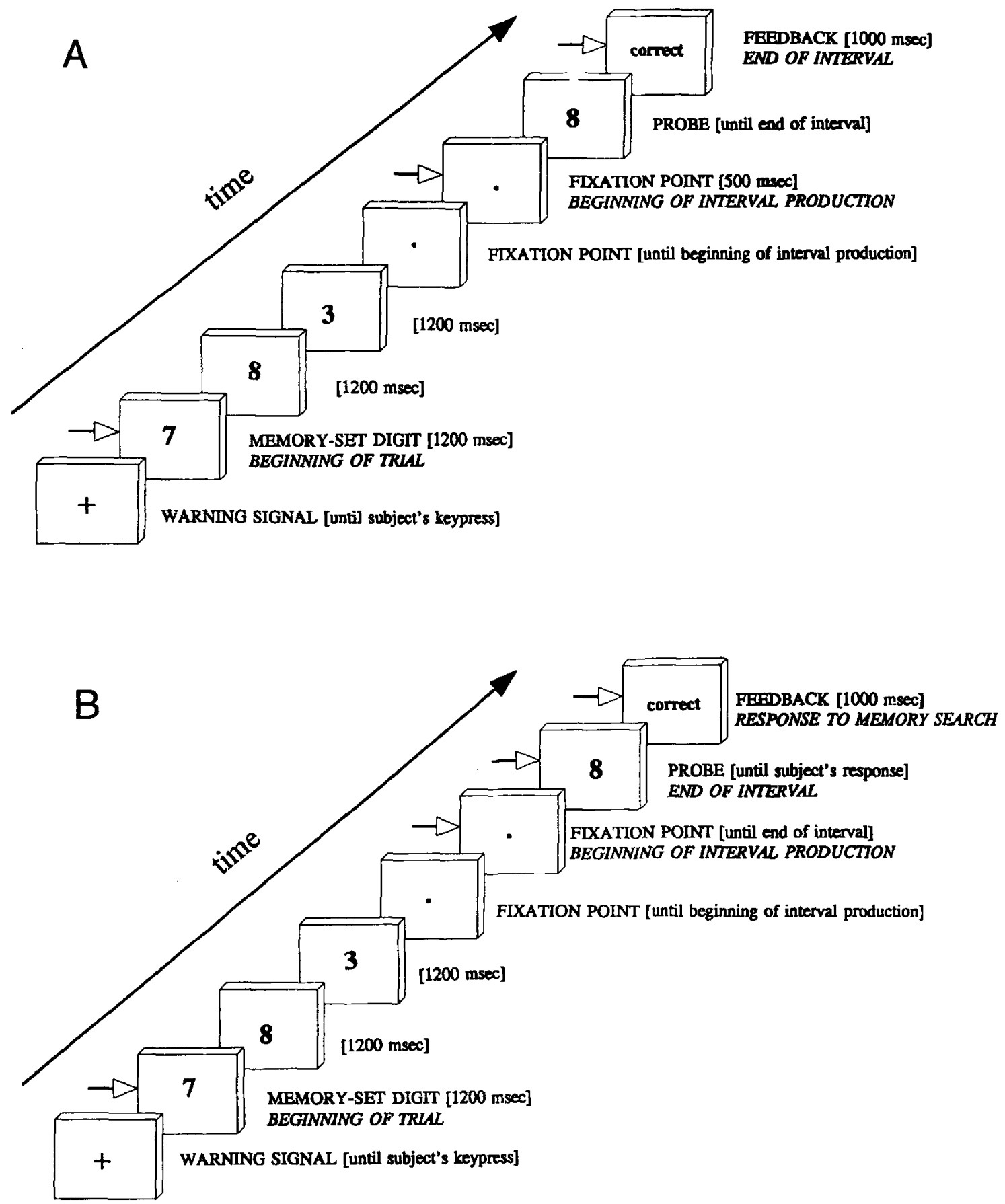

Figure 1. (A) Memory search and temporal production: a trial sequence when the probe is presented 500 msec after the beginning of the temporal production. Visual displays are described in capital letters, with their durations in brackets; subjects' keypresses are marked with short arrows and described in italics. (B) A trial sequence in Experiment 1; the probe is presented at the end of the temporal production.

temporal production accuracy was provided, while in the last block, no feedback was given. The goal of this procedure was to train subjects to produce the time interval without feedback, because in the following experimental sessions, no feedback on temporal productions would be provided in the nontemporai/temporal concurrent processing condition.
The two experimental sessions included five 48-trial blocks. The first block was a practice block, in which the subject produced temporal intervals with feedback, as in the practice sessions. This served to reset the productions around the target duration. In the next four blocks, the temporal production and memory search concurrent processing task was performed. After the warning signal, 
each concurrent processing trial, illustrated in Figure 1B, consisted of the following sequence of events: the presentation of the memory set, the beginning of the temporal production by the subject, the end of the temporal production by the subject, the presentation of the probe digit, the subject's response to the memory search, and feedback about the memory search task (correct or error). The memory-set items were presented successively, for $1,200 \mathrm{msec}$ each, without any delay between presentations. The production of the temporal interval was entirely under the subject's control and was performed by successively pressing a single key, which was located in the middle of a row of three keys. Presentation of the probe digit followed the end of temporal production immediately. The subject responded to the memory search task by pressing one or the other of the two side keys: the key on the left if the probe did not belong to the memory set (negative trial), the key on the right if it was a member of the set (positive trial).

Two responses were collected: the time interval produced by the subject, and the response time for the memory search task. The instructions were to reproduce as closely as possible the target interval produced during the practice sessions and the practice block of the experimental sessions, and then to respond to the memory search task as soon as the probe digit was presented. No particular emphasis was put on the speed of the response to the memory search task, since the aim of the experiment was to test the effect of maintaining the memory set on concurrent time estimation. Therefore, accuracy in the memory search task was more important in this perspective: it reflected that the subject did actually maintain the load in memory while producing the interval.

The relative frequency of positive and negative trials was equal within a block. The memory-set size varied from trial to trial and was balanced within a block. An experimental session included a total of 192 experimental trials, so that a total of 16 positive trials and 16 negative trials for each set size was performed in a session. There were two experimental sessions, so that each subject performed 384 trials defined by the factorial combination of the variables memory load and positive/negative trials.

The measures (temporal intervals and response times) were taken to the nearest millisecond. The temporal intervals and response times on trials on which an error was made in the memory search task were eliminated from the analyses, since it could not be certain, in such cases, that retention of the memory load had actually taken place during the temporal production. From the two data sets (temporal intervals and response times), $6.48 \%$ of the data were thus removed. The means and standard deviations of the temporal intervals and response times were computed for each subject, and any responses more than four standard deviations from the means were discarded; 12 outliers were eliminated in the response time data set, and 19 in the temporal production data set. The data from the two experimental sessions were then averaged to get, for each subject, a mean temporal interval and a mean response time for each level of memory load (1-6) and each type of trial (target present-positive and target absent-negative). Repeated measures analyses of variance (ANOVAs) were carried out on mean temporal intervals and mean response times, with set size and target presence/absence as factors (randomized block factorial design, using a nonadditive model; Huynh and Feldt's adjustment factor (e) was applied to degrees of freedom, as is recommended with repeated measures designs (Kirk, 1982). The level of statistical significance was set at at $p<.05$.

Mean temporal intervals produced with and without concurrent processing were compared. Thus, the mean and standard deviation of temporal productions without feedback, in the last block of the practice sessions, were computed for each subject, and productions more than four standard deviations from the mean were discarded (there was one outlier). These means were compared with the mean temporal intervals from the experimental sessions, pooled over all memory load and type of trial levels, in a $t$ test for dependent samples.
Error data were averaged in the same manner as were temporal productions and response times. Error rates in each combination of set size and positive/negative trial were computed for each subject over the two experimental sessions. Repeated measures ANOVAs were performed on these individual mean error rates, with set size and positive/negative trials as factors.

\section{Results and Discussion}

Temporal intervals. Mean temporal intervals in each combination of memory load level (set size) and positive/negative trial over all subjects are shown in Figure 2. The effect of the main factors memory load and positive/negative trial was not significant, nor was there a significant interaction between these factors. Varying the memory load had no effect on the duration of the produced interval. Contrasted with results of previous studies showing an interference between memory search and temporal production, this suggests that the interference, in these studies, was due to comparison of the probe with the memorized items.

The $t$ test revealed that mean temporal intervals produced in the concurrent processing condition $(M=$ $2,197 \mathrm{msec})$ were significantly longer than mean temporal intervals without feedback produced singly $(M=$ $1,701 \mathrm{msec})[t(6)=3.74, p<.01]$. This difference may reflect an interfering effect of rehearsing the memorized items to maintain them in memory during the interval duration. This effect appears to be completely independent of the memory load itself - that is, of the number of items to rehearse. However, this is confounded with a possible general cost of timesharing between temporal and nontemporal tasks, so that it is difficult to conclude on the basis of this single experiment.

Response times. As shown in Figure 3, response times increased with memory load $[F(5,30)=9.49, p<$ $.009, \epsilon=.29]$. Neither the effect of positive/negative trial, nor the interaction of memory load $\times$ positive/ negative trial was significant. The mean slope of response time as a function of memory-set size was

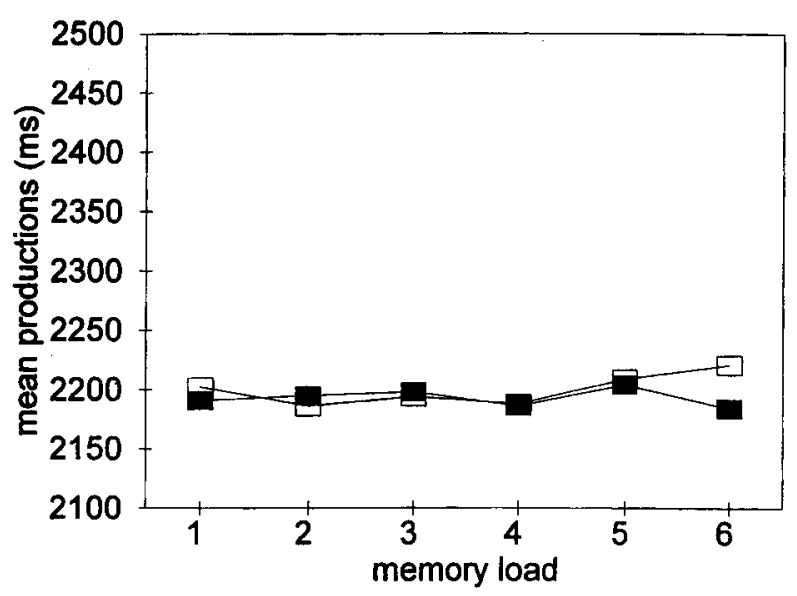

Figure 2. Mean temporal production as a function of memory load in Experiment 1 for positive trials (filled squares) and negative trials (empty squares). 


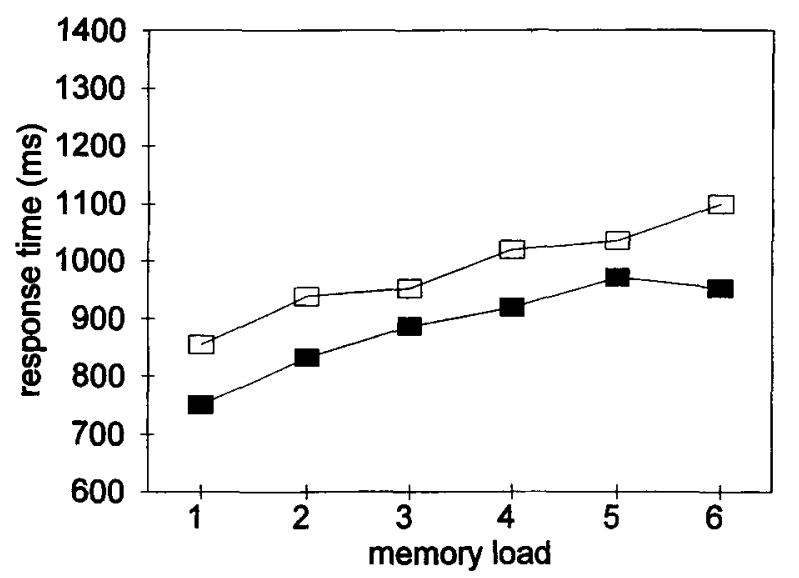

Figure 3. Mean response time as a function of memory load in Experiment 1 for positive trials (filled squares) and negative trials (empty squares).

$43 \mathrm{msec}$, a value quite close to the usual slopes of reaction time observed in memory scanning experiments with digits as members of the memory set. This suggests that the mental comparison of the target with members of the memory set was efficiently carried out after the temporal-interval production.

In the three practice sessions, the mean temporal interval produced in the first four blocks with feedback, averaged over subjects, was $2,005 \mathrm{msec}(S D=$ $256 \mathrm{msec}$ ). In the last block of these sessions - that is, in trials of temporal production without feedback - the mean produced interval was $1,701 \mathrm{msec}(S D=$ $252 \mathrm{msec}$ ). In the first block of the two experimental sessions, temporal intervals produced with feedback averaged $1,968 \mathrm{msec}(S D=208 \mathrm{msec})$. These data can be taken as evidence that subjects were able to produce relatively precise and stable time intervals around the target duration.

Errors. The mean percent error rates for each memory load and type of trial (positive/negative) are listed in Table 1. The mean percent error rate was computed for each subject, memory load, and positive/negative trial condition. These rates were subjected to a repeated measures ANOVA with the same structure as that used for temporal intervals and response times. The only effect

Table 1

Experiment 1: Mean Percent Error Rates for Each Memory-Set Size (Memory Load) in Trials with Target Present (Positive) and Target Absent (Negative)

\begin{tabular}{ccc}
\hline Memory-Set Size & Positive Trials & Negative Trials \\
\hline 1 & 0.90 & 2.67 \\
2 & 3.10 & 5.39 \\
3 & 6.26 & 2.69 \\
4 & 8.06 & 5.81 \\
5 & 10.71 & 7.14 \\
6 & 15.63 & 9.37 \\
\hline
\end{tabular}

that appeared to be significant was the effect of memoryset size on error rates $[F(5,30)=4.95, p<.03, \epsilon=.39]$. These results are consistent with those obtained with response times. They confirm that the memory-set size constitutes a load affecting the performance. Besides, if one assumes that rehearsal of items takes place during the time interval production, the amount of rehearsal per item would be inversely related to memory-set size. This could explain why performance in recognition deteriorates with increasing set size.

The overall error rate was $6.5 \%$. This is higher than error rate when memory search was completely interpolated in time production, which was below 3\% (see Fortin et al., 1993, Experiment 1). This difference may be due to the additional constraint of maintaining the load during the whole interval duration in the present experiment. This was unnecessary in Fortin et al.'s Experiment 1 , since the probe was presented $500 \mathrm{msec}$ after the beginning of temporal interval. Thus, subjects may have used a strategy, to maintain the load in memory, that led to more errors in probe detection. For example, subjects could have rehearsed the memorized items at a fixed rate to enhance the accuracy in producing the interval. Such a strategy might lead to less frequent covert repetition of the items than would occur when the subjects had the probe available during the temporal production interval. This strategy would be an indication of some influence of memory search on time estimation, since subjects would then maximize their performance on the time production task at the expense of performance on the memory search task.

The results of Experiment 1 show that temporal production does not vary when a concurrent memory load is manipulated. The results suggest further that active processing, such as the comparing of a probe with memorized items and, possibly, rehearsing these items, does. The distinction between storage and active processing is often used to characterize the concepts of short-term memory and working memory: "Working memory has traditionally been thought of as the part of the memory system where active information processing takes place.... short-term memory is usually taken to mean a passive storage system for item information, whereas working memory also contains control processes because they also require memory capacity" (Chase \& Ericsson, 1982, p. 40). Although there is a clear overlap between the concepts of short-term memory and working memory, since they both refer to the temporary holding and manipulation of information, generally their use follows the preceding distinction. Furthermore, different codes (e.g., phonological, visual, semantic) are assumed to be processed by distinct modules in working memory research. Modules can be modality specific, as in Baddeley and his colleagues' model, which includes two distinct modules for handling speech-based and visual or spatial material (Baddeley, 1986; Baddeley \& Hitch, 1974), or they can be dedicated to different types of processing (e.g., visual, auditory, phonological, lexical, se- 
mantic), as in Schneider and Detweiler's (1987) connectionist model.

In the next two experiments, the interference between time production and processes thought to be under working memory control was investigated. Two different nontemporal tasks were combined with a temporalinterval-production task. The nontemporal tasks, rhyming judgments (Experiment 2) and mental rotation (Experiment 3 ), involved no storage of information, whereas they were thought to require processing in working memory. Thus, rhyming judgments on visual stimuli would require setting up verbal-phonological representations of items and then comparing these representations in working memory (Brown, 1987), while a mental rotation task would involve visuospatial processing in working memory (Logie \& Salway, 1990). An effect of manipulating the amount of processing in these tasks on temporal production would confirm that a memory load is not essential for producing an interference in concurrent temporal and nontemporal processing.

In addition to a concurrent processing condition of temporal production and nontemporal processing in working memory, the nontemporal tasks were performed alone, in a reaction time condition. This measure provided an independent index of the difficulty level of these tasks, with difficulty being defined as response rate (Kantowitz \& Knight, 1978). Previous studies had shown that reaction times, with the nontemporal tasks used in Experiments 2 and 3, were longer than reaction times with the memory scanning task as used in Experiment 1 . To allow interpolation of the nontemporal task in the temporal-interval production, the target interval was $4 \mathrm{sec}$ in Experiments 2 and 3.

\section{EXPERIMENT 2}

A rhyming judgment task was performed, in Experiment 2, in two main conditions: a reaction time control condition, and a concurrent processing condition, in which the rhyming judgment was performed during production of a temporal interval. The subject had to judge whether a word rhymed or not with one or the other of a group of syllables - mostly nonsense syllables - the load being varied in the rhyme judgment with the number of syllables presented.

\section{Method}

Subjects. Ten subjects, 7 males and 3 females, between 19 and 31 years old (mean age, $23 ; S D=2.0$ ) took part in this experiment. They were mostly graduate and undergraduate students at Université Laval. They were paid $\$ 5$ per session for their participation.

Apparatus and Stimuli. Stimulus and feedback presentations as data collection were controlled by a PC-compatible computer running MEL software. The visual stimuli were displayed on an IBM VGA color monitor, with a $20 \times 27 \mathrm{~cm}$ screen. The responses (temporal productions, and speeded responses in the reaction time condition) were given by pressing either the left or the right of three keys on a response box (see Procedure). Subjects, seated alone in a sound-attenuated test chamber, participated individually.
Each visual display was composed of one word and of syllables, displayed simultaneously in lowercase letters on a single line. The subjects were native French speakers; the words were in French. The word always included two syllables and was displayed with one to five syllables. When fewer than five syllables were presented, the remaining locations were filled with masks. There were also two additional masks on each side of the set of syllables, so that when five syllables were displayed, the set was bounded by masks. Two sets of displays were constructed one set in which one of the syllables rhymed with the word (rhyme condition), and one set in which none of the syllables rhymed (nonrhyme condition). For each rhyming condition, and for each number of syllables (one to five), 50 displays were constructed. Examples of displays for each of these conditions are listed in the Appendix. The last syllable of the word and the rhyming syllable were, as much as possible, visually different; that is, they consisted of different combinations of letters, to avoid subjects' responding on the basis of visual examination of stimuli. In the rhyme condition, the target - the rhyming syllable-was presented equally often in each of the five possible locations. The visual angle of the whole presentation was about $0.43^{\circ}$ in height and $10^{\circ}$ in width. The letters were white on a black background.

Procedure. One group of 5 subjects was tested in the reaction time condition, and another group of 5 subjects was tested in the concurrent processing condition. In the reaction time condition, the subjects performed the rhyme-judgment task with the instruction to respond as quickly as possible while minimizing errors. In the concurrent processing condition, the subjects first practiced producing a given time interval during practice sessions; this was used to stabilize the time production performance. After the practice sessions, in the experimental sessions the subjects were instructed to produce the target interval as closely as possible while simultaneously performing a rhyme-judgment task.

In the reaction time condition, 5 subjects were tested in three experimental sessions consisting of four 50-trial blocks. In a block, half of the trials were rhyme trials, and half were nonrhyme trials. The number of syllables varied from trial to trial and was balanced within a block. At the beginning of each trial, the subject viewed a fixation point, with one of his/her hands resting on the response box. The subject was instructed to put three fingers of the same hand on the three keys of the response box. When ready to begin the trial, the subject pressed the middle key. The fixation point reappeared for $500 \mathrm{msec}$, and after this delay it was followed by a stimulus presentation. The subject responded as fast as possible by pressing the key located on the right if the word and the syllable rhymed, and by pressing the left key if they did not rhyme. Immediately after the response, visual feedback informed the subject whether the response was correct. The feedback remained present for $1,000 \mathrm{msec}$; this was followed by the fixation point presentation indicating the beginning of the next trial. Sessions lasted between 30 and $45 \mathrm{~min}$.

In the concurrent processing condition, subjects first practiced producing a 4-sec interval during three practice sessions, which included five 50-trial blocks. At the beginning of a trial, a fixation point was present. When ready, the subject began the interval by depressing the middle key. The fixation point remained present for $500 \mathrm{msec}$ and was then replaced by an arrow indicating to the subject on which key (right or left) to press when he/she estimated that the interval had elapsed, a procedure aimed at familiarizing the subject with the dual response. In the first four blocks, visual feedback for the temporal production task was provided, informing the subject if the produced interval was under, over, or within a 400msec window centered on the 4-sec standard. In the fifth and last block, no feedback was provided. After the three practice sessions, there were three experimental sessions. In these sessions, the subject was run first in a 50-trial block of temporal production with feedback, followed by four 50-trial blocks of temporal production and rhyme judgment. In the experimental trials, the sub- 
ject began the interval by pressing the middle key. A fixation point appeared for $500 \mathrm{msec}$, followed immediately by the stimulus presentation. The display, a word followed by syllables on a single line, remained present until the subject ended the interval by pressing the right or the left key to indicate whether the word did or did not rhyme with one or the other of the syllables. (Note that the structure of the trial is similar to that shown in Figure 1A, with the exception that there is no memory set presented before the temporal production: the display, word and syllables, is presented $500 \mathrm{msec}$ after the beginning of the interval production and remains present until the end of the interval.) Visual feedback was given, informing the subject whether or not the response was correct. No feedback was provided on the temporal production performance. As in the reaction time condition, rhyme and nonrhyme trials and the number of syllables varied from trial to trial and were balanced within a block. Experimental sessions lasted approximately $45 \mathrm{~min}$.

Prior to the experiment, each subject was given an initial introductory session to ensure familiarity with the apparatus, stimuli, and experimental procedure. In the practice and experimental sessions of both the reaction time and the concurrent processing conditions, there was a 30 -sec rest between blocks.

The measures-reaction times in the reaction time condition, and temporal intervals in the concurrent processing conditionwere taken to the nearest millisecond. In each of the two main conditions, reaction time and concurrent processing, each subject performed 600 experimental trials defined by the factors number of syllables and rhyme/nonrhyme. In the reaction time condition, $3.50 \%$ of the data were removed from the analysis because there was an error in the rhyme judgment in these trials. In the concurrent processing condition, temporal intervals in trials where an error occurred in the rhyme-judgment task-that is, $2.77 \%$-were eliminated from the data set. Mean and standard deviation of reaction times in the reaction time group and of temporal intervals in the temporal production group were computed for each subject. Data more than four standard deviations from the means were discarded; that represented eight observations in the reaction time data set and six temporal intervals. Data were averaged to get, for each subject, a mean reaction time or a mean temporal interval for each condition combinationthat is, number of syllables (one to five) and rhyme/nonrhyme. Repeated measures ANOVAs were carried out on mean temporal intervals and mean reaction times with the same condition combination. The level of statistical significance was set at at $p<.05$.

Mean temporal intervals produced with and without concurrent processing were compared. Individual means and standard deviations of temporal productions without feedback were computed as in Experiment 1, and three outliers (more than four standard deviations from the mean) were discarded. These means were compared with the mean temporal intervals of the experimental sessions, pooled over all numbers of syllables and rhyme/nonrhyme conditions, in a $t$ test for dependent samples.

\section{Results and Discussion}

Reaction times. The mean reaction times averaged over subjects are graphed as a function of the number of syllables in Figure 4. With reaction time data, the number of syllables main effect was significant $[F(4,16)=$ $17.20, p<.009, \epsilon=.29]$. The effect of rhyme/nonrhyme condition did not reach significance, but the interaction of number of syllables $\times$ rhyme/nonrhyme did $[F(4,16)=12.47, p<.0001, \epsilon=.99]$; the effect of increasing the number of syllables was stronger in the nonrhyme condition than in the rhyme condition. The

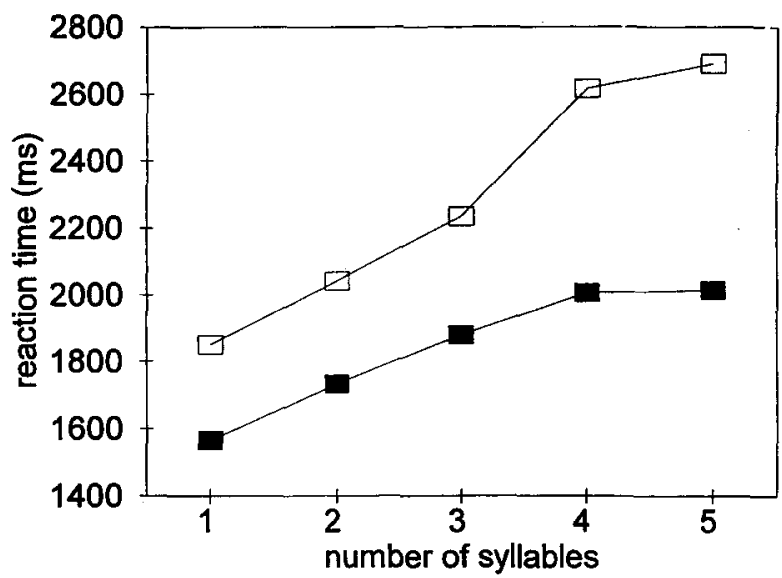

Figure 4. Mean reaction time as a function of number of syllables in Experiment 2 for rhyme trials (filled squares) and nonrhyme trials (empty squares).

slopes of the mean reaction times were $116.5 \mathrm{msec}$ and $225.6 \mathrm{msec}$ in the rhyme and nonrhyme conditions, respectively. The overall $1: 2$ ratio between the slopes suggests that the search for a rhyming syllable is selfterminating; that is, it ends when a rhyming syllable is found.

Temporal intervals. In Figure 5, the mean temporal intervals are shown as a function of the number of syllables. The effect of number of syllables $[F(4,16)=4.18$, $p<.03, \epsilon=.73$ ] was significant, suggesting that some processing, in the rhyme-judgment task, interfered with time estimation. The effect of rhyme/nonrhyme main condition was also significant $[F(1,4)=36.20, p<$ $.004]$ but the interaction of number of syllables $X$ rhyme/nonrhyme was not. The slopes of mean temporal intervals as a function of the number of syllables were $11.0 \mathrm{msec}$ in the rhyme condition and $29.6 \mathrm{msec}$ in the nonrhyme condition. The proportional increase of tem-

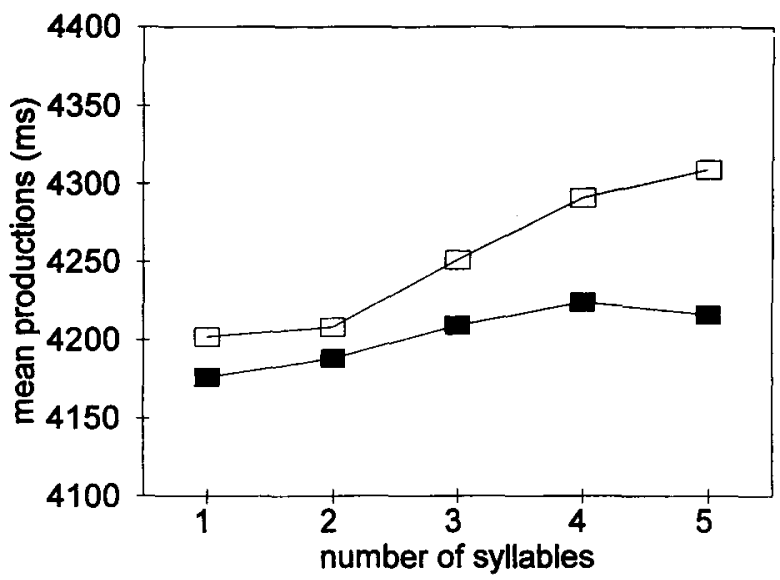

Figure 5. Mean temporal production as a function of number of syllables in Experiment 2 for rhyme trials (filled squares) and nonrhyme trials (empty squares). 
poral productions with the number of syllables strongly suggests that the lengthening of the temporal intervals is actually related to the processing of the syllables.

In the concurrent processing condition, mean temporal intervals $(4,228 \mathrm{msec})$ were longer than mean temporal intervals without feedback produced singly $(3,935 \mathrm{msec})[t(4)=3.32, p<.05]$. This difference may be due to a general load induced by the concurrent nontemporal task. In Experiment 1, longer temporal intervals in the concurrent processing condition could be attributed to a general cost of a concurrent processing and/or to rehearsal of memory-set items. Since there was no memory load in Experiment 2, the lengthening of intervals cannot be explained by rehearsal. However, it is possible that maintaining a load in memory and rhyme judgment share some specific processes (e.g., related to processing verbal material) that could have produced the overall lengthening in both concurrent processing conditions.

In the three practice sessions, the overall mean temporal interval produced with feedback was $4,065 \mathrm{msec}$ $(S D=463 \mathrm{msec})$. In trials of temporal production without feedback, the mean produced interval was $3,935 \mathrm{msec}(S D=541 \mathrm{msec})$. In the first block of the three experimental sessions, temporal intervals produced with feedback averaged $4,058 \mathrm{msec}(S D=$ $517 \mathrm{msec}$ ). As in Experiment 1, these data show that practice allowed subjects to produce relatively precise and stable time intervals around the target duration.

Errors. In Table 2, the mean percent error rates for each condition-reaction time and temporal production-are given for each combination of number of syllables $\times$ rhyme/nonrhyme. The mean percent error rate was computed for each subject and each condition combination. These rates were submitted to a repeated measures ANOVA as reaction times and temporal productions. In the reaction time condition, the effect of number of syllables was significant $[F(4,16)=5.06, p<.008$, $\epsilon=1.81$ ], confirming that increasing the number of syllables to process also increases the difficulty of rhyme judgments. The effect of rhyme/nonrhyme was not significant, but the interaction of number of syllables $X$ rhyme/nonrhyme was $[F(4,16)=4.65, p<.03, \epsilon=.62]$.

In the concurrent processing condition, the number of syllables also had a significant effect on error rates $[F(4,16)=3.48, p<.03, \epsilon=1.42]$, as did the rhyme/

Table 2

Experiment 2: Mean Percent Error Rates for Each Number of Syllables in Rhyme and Nonrhyme Trials in Reaction Time and Concurrent Processing Conditions

\begin{tabular}{|c|c|c|c|c|}
\hline \multirow{2}{*}{$\begin{array}{c}\text { Number of } \\
\text { Syllables }\end{array}$} & \multicolumn{2}{|c|}{ Reaction Time } & \multicolumn{2}{|c|}{ Concurrent Processing } \\
\hline & Rhyme & Nonrhyme & Rhyme & Nonrhyme \\
\hline 1 & 2.00 & 1.00 & 2.00 & 0.34 \\
\hline 2 & 5.02 & 0.66 & 5.00 & 1.68 \\
\hline 3 & 5.66 & 0.68 & 7.00 & 0.68 \\
\hline 4 & 4.66 & 4.68 & 4.68 & 2.34 \\
\hline 5 & 4.66 & 6.00 & 3.34 & 0.68 \\
\hline
\end{tabular}

nonrhyme condition $[F(1,4)=33.00, p<.005]$. The interaction of number of syllables $X$ rhyme/nonrhyme was not significant. In the concurrent processing condition, the effects on error rates were the same as those affecting temporal productions.

The main observation, in the present experiment, concerns the correspondence between the functions of reaction time and temporal productions versus the number of syllables. Reaction times vary systematically with the number of syllables in a rhyme-judgment task and temporal productions also vary with increasing the number of syllables if the rhyming task is interpolated in the production. This suggests that some operations, in the rhyming judgment, interfere with time estimation. The fact that temporal productions lengthen with increasing the number of syllables to process suggests that some nontemporal processing in the nontemporal task could interrupt pulse accumulation at some point in the time estimation task. These operations are likely to be part of the processing of the syllables, since the lengthening is positively related to the number of syllables.

Fortin et al. (1993) have shown that a visual search task (e.g., searching for a green $\mathrm{T}$ among brown Ts and green Xs) interpolated in temporal production did not interfere with time estimation. This suggests that the interference, in the present experiment, is not related to the visual processing involved in the rhyming judgment.

In the same paradigm with a nontemporal task interpolated in temporal production, the results suggested that the interference from nontemporal search tasks on temporal production is specifically dependent on processing short-term stored information (Fortin et al., 1993). Now the results of Experiment 2 show a clear interference between time production and a nontemporal task involving no short-term retention as such. The visual stimuli on which the judgment is made remain present until the response is given. This does not imply, however, that the rhyme-judgment task does not require some form of processing in a temporary memory system. Actually, rhyming judgment has been investigated as a working memory task requiring a phonological or articulatory component in working memory (Baddeley \& Lewis, 1981; Besner, Davies \& Daniels, 1981; Brown, 1987; Wilding \& White, 1985). Thus, in the rhyming task, the subject would have to to set up a phonological representation, which would then be compared with the phonological representation of the last syllable of the word. Therefore, the setting up and/or the comparing of the phonological representations corresponding to each syllable could be the interfering processes.

Although the mean reaction time slope $(172 \mathrm{msec})$ was much steeper in the present experiment than in preceding similar experiments with an interpolated memory search task (about $35 \mathrm{msec}$ in Fortin \& Rousseau, 1987, and Fortin et al., 1993), the slopes of the temporal production functions, when these nontemporal tasks were interpolated in temporal production, were surprisingly similar: about $25 \mathrm{msec}$ in the preceding experiments and $20 \mathrm{msec}$ 
in the present experiment. High reaction time slopes were expected with rhyming judgments, since the visual operations needed in reading add to the total duration of the nontemporal task. However, it could be the case that these visual operations, whose duration is necessaril included in a measure as global as reaction time, do not interfere with time estimation. The lengthening of 20 $25 \mathrm{msec}$ per item across experiments could therefore pinpoint a basic component of the scanning process in the nontemporal tasks, a component that is incompatible with estimating time and that would possibly use a phonological component of working memory.

\section{EXPERIMENT 3}

In this experiment, a task known to require mental rotation was executed in a reaction time condition and then concurrently with a temporal production task. Performance in a mental rotation task was found by Logie and Salway (1990) not to be affected by concurrent articulatory suppression, whereas a clear disruption in mental rotation was induced by performing a spatial suppression task. This suggests that mental rotation is performed independently of the phonological component of working memory, requiring instead a visuospatial component. Interpolating a mental-rotation task in a temporal production allows one to test specifically whether such visuospatial operations, in working memory, interfere with time estimation. As with rhyme judgments, this task involves no memory load: stimuli-pairs of polygons - are presented visually and remain present for the whole duration of the concurrent processing task. The left polygon is in a constant position, considered as the standard upright position. The right one is an exact copy or a mirror version of the polygon on the left. Subjects are requested to decide whether the two polygons are the same or different. Polygons on the right are shown in six angular disparities relative to the left polygon $\left(0^{\circ}, 60^{\circ}, 120^{\circ}, 180^{\circ}, 240^{\circ}\right.$, and $\left.300^{\circ}\right)$.

As in Experiment 2, two main conditions were included: mental rotation in a reaction time condition, and mental rotation combined with time interval production in a concurrent processing condition. On the basis of previous data on mental rotation with polygons (e.g., Folk \& Luce, 1987), overall reaction times were expected to be comparable to those in rhyming judgments in Experiment 2. Since in the concurrent processing condition the target interval in which nontemporal processing was interpolated was the same as in Experiment 2-. that is, $4 \mathrm{sec}$-it should be possible to compare directly the effect of nontemporal processing in working memory with time estimation.

\section{Method}

Subjects. Ten subjects, 7 females and 3 males, between 19 and 23 years old (mean age, $21 ; S D=1.7$ ) took part in this experiment. They were mostly undergraduate and graduate students at Université Laval. They were paid \$5 per session for their participation. None had participated in preceding experiments.

Stimuli and Apraratus. The stimuli used in the mental rotation task were eight standard polygons selected from the set provided by Vanderplas and Garvin (1959). Five levels of complexity, defined by the number of points determining inflections on the perimeter of the form, were represented. One 6-pointed polygon, two 8-pointed, two 12-pointed, two 16-pointed, and one 24pointed polygon were selected. These eight standard polygons were respectively assigned the following codes: la (6-pointed), 2a and $3 \mathrm{a}$ (8-pointed), $4 \mathrm{a}$ and $5 \mathrm{a}$ (12-pointed), $6 \mathrm{a}$ and $7 \mathrm{a}$ (16-pointed), and $8 \mathrm{a}$ (24-pointed). The mirror versions of these polygons were respectively assigned the codes $\mathrm{lb}$ to $8 \mathrm{~b}$. These forms were scanned from Sidowski's (1966, pp. 374-375) presentation of the forms with a scanner (Lightning Scan Pro 256). Pairs of polygons were then constituted, with a normal or a mirror version of a polygon in an upright position on the left, and on the right, either a normal or a mirror version of the same polygon, in one of the six possible orientations $\left(0^{\circ}, 60^{\circ}, 120^{\circ}, 180^{\circ}, 240^{\circ}, 300^{\circ}\right)$. With a given polygon and its mirror version, four pairs could be constitutedfor example, $1 a-1 a, 1 a-1 b, 1 b-1 a, 1 b-1 b$. Furthermore, for each of these pairs, the polygon on the right could appear in one of the six orientations, thus giving a set of 24 pairs with each polygon. With the eight polygons, a total of 192 pairs were constructed. Examples of pairs, constituted with each of the eight standard polygons used in the experiment, are illustrated in Figure 6.

Pairs of polygons were printed on white labels (Avery $15.2 \times$ $10.2 \mathrm{~cm}$ ). The labels were mounted on cards of the same dimension - that is, $15.2 \times 10.2 \mathrm{~cm}$. The stimuli were displayed with a Gerbrands four-channel tachistoscope, at a viewing distance of $72 \mathrm{~cm}$. The visual angle subtended by the display was $7.2^{\circ}$ in height and $10.7^{\circ}$ in width. The polygons subtended approximately $1.9^{\circ}$ in height and $1.9^{\circ}$ in width.

Temporal productions and responses to the mental rotation task were given by pressing the left or the right key of three keys on a response box.

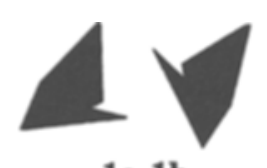

1a-1b

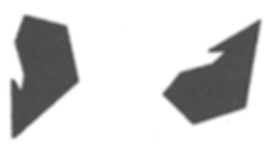

$2 a-2 b$

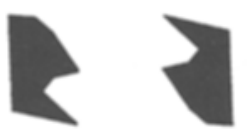

3a-3a

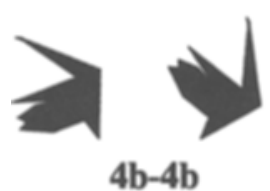

Figure 6. Examples of pairs of polygons used in Experiment 3. The pairs shown are, following the codes defined in the text, $1 \mathbf{a}-1 \mathbf{b}, \mathbf{2 a - 2 b}$, $3 a-3 a$, and $4 b-4 b$ in the left column, and $5 b-5 b, 6 a-6 b, 7 a-7 b$, and $8 b-8 a$ in the right column. 
Procedure. Five subjects were tested in the reaction time condition, five others in the concurrent processing condition. The mental rotation task was performed singly in the reaction time condition, with the instruction that subjects should respond as soon as possible. In the concurrent processing condition, 5 subjects were run through temporal production practice sessions, followed by experimental sessions in which time production and mental rotation were performed concurrently. In the reaction time condition, three experimental sessions included four 48-trial blocks. In the concurrent processing condition, the three temporal production practice sessions (temporal production with feedback) included five 48-trial blocks. In the three following experimental sessions, a first 48-trial block of temporal production with feedback was followed by four 48-trial blocks of concurrent temporal production and mental rotation, without feedback on temporal production performance, but with feedback on mental rotation.

In each of the 48-trial blocks in which mental rotation was performed, the eight polygons used as stimuli were presented in pairs. In a single block, the six possible orientations of the polygon on the right were represented. In half the trials of a block, the correct response was "same"; that is, the polygons (either normal or mirror) of the pair were identical. In the other half of the trials of the block, the polygons were different. The order of presentation of the pairs was chosen at random by the computer. All possible pairs of the polygons $(1 a-1 a, 1 a-1 b, 1 b-1 a, 1 b-1 b, \ldots 8 b-8 b$; i.e., each pair presented with the six orientations) were presented in a session, for a total of 192 trials. These trials constituted the four 48-trial blocks in the experimental sessions of the reaction time condition, and also the four last 48-trial blocks in the concurrent processing condition-that is, the blocks in which temporal production and mental rotation were performed concurrently.

In the reaction time condition, three adjacent keys were used. A trial had the following structure: When ready, the subject pressed the key on the right. Then a fixation point appeared at the center of the screen for $1,000 \mathrm{msec}$. The fixation point was followed by the pair of polygons, which remained present until the subject pressed one of the two response buttons. The subject was instructed to press the middle key as soon as possible when the polygons were identical, and the key on the left when they were different. Visual feedback (SAME, DIFFERENT) was presented immediately after the subject's response for $1,000 \mathrm{msec}$. Each subject performed three experimental sessions, each of which included four 48-trial blocks, with 30 -sec rests between blocks. The sessions lasted between 30 and $40 \mathrm{~min}$.

In the concurrent processing condition, trials in the three practice sessions had the same structure as in Experiment 2: first the subject viewed the fixation point; then the subject began the temporal interval by depressing the middle key; the fixation point remained for $500 \mathrm{msec}$, and was then replaced by an arrow indicating to the subject on which key (right or left) to press when he/she estimated that the interval had elapsed. In the first four blocks, visual feedback informed the subject whether his/her production was correct, within a 400-msec window centered on the 4-sec standard. In the fifth and last block, no feedback was provided. In the three experimental sessions, the first block of temporal production with feedback was followed by five blocks of temporal production and mental rotation. Orientation of rotated stimulus and type of pair (same-different) were varied from trial to trial. In a block of trials, all six angular orientations were used an equal number of times, with half the pairs being constituted of same polygons, and half of different polygons. The other aspects of the procedure were the same as in Experiment 2.

In the three following experimental sessions, there was, as in Experiment 2, a first block of temporal production with feedback, followed by four blocks in which the subject performed the concurrent processing task. The experimental trials were as in Experiment 2-that is, fixation point for $500 \mathrm{msec}$, followed immediately by the display presentation. The display-a pair of polygons-remained present until the subject ended the interval by pressing the right or the left key to indicate whether the pairs included identical or different polygons. Visual feedback was provided, informing the subject whether the polygons were the same or different. No feedback was provided on the temporal production performance. The experimental sessions lasted approximately $45 \mathrm{~min}$.

In each of the main conditions, reaction time and concurrent processing, each subject performed 576 experimental trials defined by the factorial combination of the variables orientation and type of pair (sameldifferent). Measures were recorded to the nearest millisecond, with removal of trials in which an error was made in the nontemporal task: $6.53 \%$ in the reaction time data set, and $5.80 \%$ in the temporal interval data set. Means and standard deviations of reaction times and of temporal intervals were computed for each subject. Data more than four standard deviations from the individual means were discarded: four observations in the reaction time data set, and five temporal intervals. A mean reaction time or a mean temporal interval for each orientation was computed for each subject: these means were subjected to repeated measures analyses of variance with orientation $\left(0^{\circ}, 60^{\circ}, 120^{\circ}, 180^{\circ}, 240^{\circ}\right.$, and $300^{\circ}$ ) and type of pair (sameldifferent) as factors. The level of statistical significance was set at at $p<.05$.

The mean temporal intervals produced singly without feedback were compared with the mean temporal intervals in the concurrent processing condition. Individual means and standard deviations of temporal productions of the last block of practice sessions were computed. There was no outlier; that is, there was no temporal production more than four standard deviations from the mean. These means were compared with the mean temporal intervals of the experimental sessions, pooled over all orientation and same/different conditions with a $t$ test for dependent samples.

\section{Results and Discussion}

Reaction times. The mean reaction times averaged over correct responses are shown in Figure 7 (the point, at $360^{\circ}$, duplicates the point at $0^{\circ}$ ). A strong effect of angular disparity was found $[F(5,20)=19.62, p<.0007$, $\epsilon=.41]$. Responses in the same condition were significantly faster than those in the different condition $[F(1,4)=$ $8.66, p<.04]$. It can also be seen, in Figure 7 , that the pattern of results across the different orientations was different for same and different trials $[F(5,20)=4.85$,

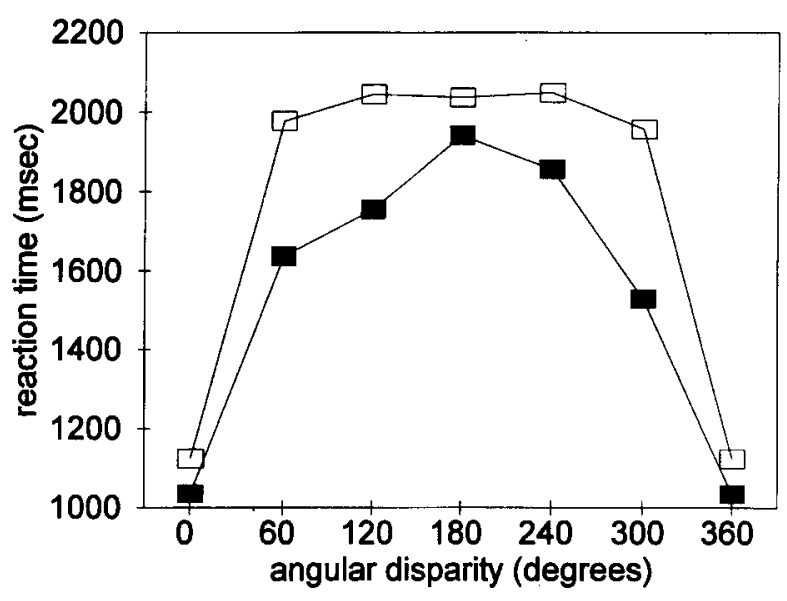

Figure 7. Mean reaction time as a function of angular disparity between polygons in Experiment 3 for same trials (filled squares) and different trials (empty squares). 
$p<.01]$ : in the same condition, the proportionality of reaction times with angular disparity from the upright standard position is clearly stronger.

This is not uncommon in research on mental rotation (Cooper \& R. N. Shepard, 1982; J. Metzler \& R. N. Shepard, 1974), especially in a two-stimulus task as with pairs of polygons; actually, it seems that in these conditions, subjects are sometimes able to base their negative responses (i.e., different responses) on short-cut nonrotational strategies, particularly when the number of structurally different objects and orientations presented is small (S. Shepard \& D. Metzler, 1988). This is why in many cases, data from the different condition are not included in the analyses (e.g., D. Shepard \& J. Metzler, 1971; S. Shepard \& D. Metzler, 1988). Because in the present experiment the number of orientations and of objects (polygons) was relatively high, the different trials were included. However, the fact that reaction times are less proportional to angular disparity in the different condition suggests that on some of these trials, nonrotational strategies could actually have been used by some subjects. For correct same responses, Figure 7 shows that mean reaction time increases clearly with angular difference between the polygons. This is usually taken as evidence for the use of a mental rotation strategy, which is also reported by subjects as being consciously carried out.

Temporal intervals. As with reaction times, there was a strong overall effect of angular difference between polygons to process on mean temporal intervals produced simultaneously $[F(5,20)=9.28, p<.0001, \epsilon=$ 1.42], as shown in Figure 8. There was no main effect of type of pairs (same/different), but the effect of angular disparity appeared to be different for same and different trials $[F(5,20)=3.23, p<.03, \epsilon=0.88]$. In fact, the lengthening of temporal intervals was more proportional to angular difference in the same condition than in the different condition. Since reaction time data (in this experiment as in previous ones) suggest that mental rotation is used more regularly in the same condition than in the different condition, this supports an interpretation according to which the interference between the nontemporal and temporal tasks would be induced specifically from the mental rotation component of the nontemporal task. These findings suggest that visuospatial processing in working memory interferes with concurrent time estimation even if processing in working memory does not involve short-term retention.

Although mean temporal intervals produced in the concurrent processing condition $(M=4,565 \mathrm{msec})$ were longer than intervals produced singly $(M=4,240 \mathrm{msec})$, this difference was not significant. This suggests that in Experiments 1 and 2, the interpretation following which the difference between these conditions would reflect a general cost of timesharing is incorrect. However, the interpretation of this negative result must take into account the small sample size used in this test. Actually, 5 subjects were used in each condition, so that a lack of power may explain the nonsignificant result of the $t$ test. Power

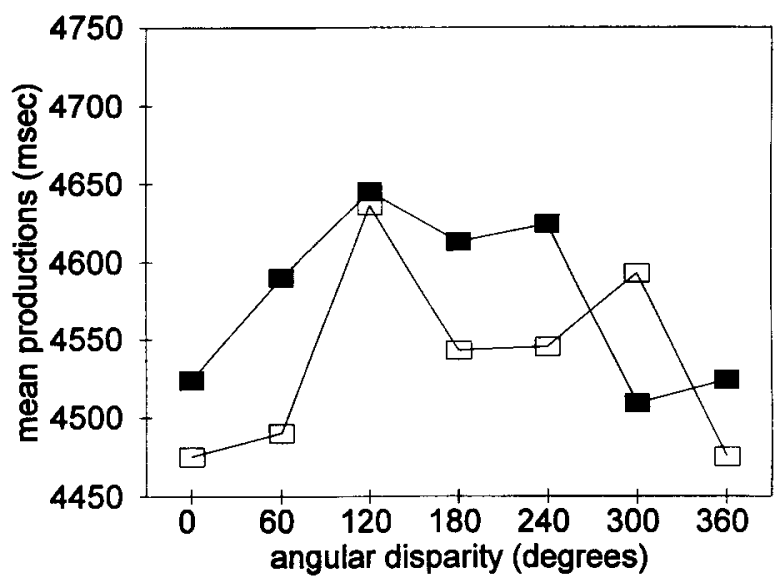

Figure 8. Mean temporal production as a function of angular disparity between polygons in Experiment 3 for same trials (filled squares) and different trials (empty squares).

to detect a medium-sized difference (.5 standard deviation of the mean for the single task condition) was estimated to be low-that is, .30 . Therefore, the possibility remains that some cost of timesharing explains the 325msec difference between the single and concurrent processing conditions.

The overall mean temporal interval produced with feedback in the three time production practice sessions was $3,971 \mathrm{msec}(S D=339 \mathrm{msec})$. In trials of temporal production without feedback, the mean produced interval was $4,240 \mathrm{msec}(S D=543 \mathrm{msec})$. In the first block of the three experimental sessions, temporal intervals produced with feedback averaged $3,979 \mathrm{msec}(S D=$ $291 \mathrm{msec}$ ). With practice, subjects thus seemed to be able to produce an interval around the target duration.

Errors. The mean percent error rates, computed for each subject and each condition, were subjected to a repeated measures analysis of variance with angular disparity and type of pairs as factors. In the reaction time condition, the only significant effect was the overall effect of angular disparity on error rates $[F(5,20)=5.79$, $p<.02, \epsilon=0.49$ ]. The mean percent error rates for all angular differences and type of pairs are listed in Table 3. Overall, error rates tend to be correlated with reaction time, suggesting that responding is based on mental rotation of stimuli.

In the concurrent processing condition, the effect of angular difference on error rates was significant $[F(5,20)=$ $5.64, p<.002, \epsilon=1.11]$. The interaction of angular difference $\times$ type of pair was also significant $[F(5,20)=$ $3.23, p<.03, \epsilon=1.28$ ]: error rates appeared to be more positively related with reaction time in the same condition than in the different condition. This appears consistent with an interpretation in terms of the use of nonrotational strategies in some different trials. Actually, S. Shepard and D. Metzler (1988) found, with mental rotation of polygons and three-dimensional stimuli, that error rates on positive trials were correlated with reaction times, increasing roughly with angular difference, 
Table 3

Experiment 3: Mean Percent Error Rates for Angular Disparity Between Polygons in Trials with Pairs of Same and Different Polygons in the Reaction Time and the Concurrent Processing Conditions

\begin{tabular}{crcccc}
\hline & \multicolumn{2}{c}{ Reaction Time } & & \multicolumn{2}{c}{ Concurrent Processing } \\
\cline { 2 - 3 } \cline { 5 - 6 } Orientation & Same & Different & & Same & Different \\
\hline $0^{\circ}$ & 0.84 & 1.26 & & 0.42 & 3.34 \\
$60^{\circ}$ & 3.74 & 2.94 & & 2.94 & 5.86 \\
$120^{\circ}$ & 4.60 & 4.58 & & 7.10 & 2.52 \\
$180^{\circ}$ & 14.58 & 7.50 & 7.10 & 8.34 \\
$240^{\circ}$ & 10.42 & 9.60 & & 5.04 & 10.00 \\
$300^{\circ}$ & 9.18 & 9.16 & 5.84 & 11.26 \\
\hline
\end{tabular}

whereas on negative trials, error rates were not systematically related to angular difference. This was again taken as evidence of the use of nonrotational strategies on some of the negative trials. It is interesting to note that this difference in the trends (i.e., the interaction), in the present experiment, reached statistical significance in the concurrent processing condition with error and with time production data. This suggests that time interval production was sensitive to the difference in the cognitive processes required in the same and different conditions, at least on a certain proportion of trials.

\section{GENERAL DISCUSSION}

Memory search was shown by Fortin et al. (1993) to interfere with time production, whereas attentiondemanding visual search produced no interference with temporal interval production. This was taken as evidence that time estimation required operating specifically in short-term memory or working memory.

In the present study, we aimed to investigate this issue further, by testing, independently, interference between temporal production and a memory load (Experiment 1), phonological processing (Experiment 2), and visuospatial processing (Experiment 3 ). The first experiment showed that manipulating a memory load had no effect on concurrent time production. In Experiments 2 and 3, temporal interval production varied systematically with concurrent nontemporal tasks involving no memory load.

In Experiment 2, time intervals produced by subjects were lengthened significantly by concurrently performing rhyme judgments, and the lengthening tended to be proportional to the number of syllables on which a judgment had to be performed. Rhyming judgments of visually presented stimuli are assumed to require that one set up phonological representations of items and then compare these representations in working memory (Brown, 1987). The proportional lengthening of temporal intervals with the number of syllables to process in the rhyming task suggests that one or both of these operations interferes with time estimation.

The interference between a task requiring phonological processing in working memory and time production suggests that time estimation may use a verbal-phonological module in working memory that could be the phonological loop in Baddeley's model or the auditory module in Schneider and Detweiler's model of working memory (Baddeley, 1986; Schneider \& Detweiler, 1987). It may be relevant to relate this with the fact that integrating counting and time estimation improves accuracy of temporal estimation (e.g., Fetterman \& Killeen, 1990; Getty, 1976; Petrusic, 1984). Counting is assumed to be a basic operation in the verbal-phonological component of working memory, and, as such, it is often used as a suppression task to demonstrate, in a concurrent processing paradigm, the use of a verbal-phonological component of working memory.

In Experiment 3, a polygon-matching task in which difficulty was manipulated with varying angular disparity between stimuli was combined with temporal production. Overall, interference proportional to angular disparity was again observed in this experiment. Since mental rotation would require operating in visuospatial working memory (Logie \& Salway, 1990), it seems that temporal interval production also requires this component of working memory.

Michon (1975) suggested that estimations of duration greater than $500 \mathrm{msec}$ were supported by short-term memory. This idea remains present in the current research on time estimation. For example, it is assumed that on-line processing of temporal information occurs most probably in working memory (Zakay, 1993). A psychophysical model of time estimation in animals includes working memory as a memory system wherein the current result of temporal information accumulation is held (Church, 1984). The present research is a systematic investigation of this issue.

The additive interference observed in the present experiments may be interpreted within a psychophysical timing model framework (e.g., Meck, 1984; Rousseau et al., 1984): processing in working memory could interrupt accumulation of temporal information (pulses) emitted from an internal source, thus lengthening the time required for the accumulation of a criterion number of pulses corresponding to the target duration. The present study suggests further that the interruption mechanism or the accumulation process is controlled by processing in working memory without use of its storage capacity.

\section{REFERENCES}

BADDELEY, A. D. (1986). Working memory. Oxford: Oxford University Press.

BadDeley, A. D., \& Hitch, G. (1974). Working memory. In G. H. Bower (Ed.), The psychology of learning and motivation (Vol. 8, pp. 47-89). New York: Academic Press.

BADDELEY, A. D., \& LewIs, V. J. (1981). Inner active processes in reading: The inner voice, the inner ear and the inner eye. In A. M. Lesgold \& C. A. Perfetti (Eds.), Interactive processes in reading (pp. 107-129). Hillsdale, NJ: Erlbaum.

Besner, D., Davies, J., \& Daniels, S. (1981). Reading for meaning: The effects of concurrent articulation. Quarterly Journal of Experimental Psychology, 33A, 415-437.

BLock, R. A. (1990). Models of psychological time. In R. A. Block (Ed.), Cognitive models of psychological time (pp. 1-35). Hillsdale, NJ: Erlbaum. 
Brown, G. D. A. (1987). Phonological coding in rhyming and homophony judgement. Acta psychologica, 65, 247-262.

Chase, W. G., \& Ericsson, K. A. (1982). Skill and working memory. In G. H. Bower (Ed.), The psychology of learning and motivation (Vol. 16, pp. 2-58). New York: Academic Press.

Church, R. M. (1984). Properties of the internal clock. In J. Gibbon \& L. Allan (Eds.), Timing and time perception (Annals of the New York Academy of Sciences, Vol. 423, pp. 566-582). New York: New York Academy of Sciences.

CoOper, L. A., \& Shepard, R. N. (1982). Mental images and their transformations. Cambridge, MA: MIT Press.

Fetterman, J. G., \& KilleEn, P. R. (1990). A componential analysis of pacemaker-counter timing systems. Journal of Experimental Psychology: Human Perception \& Performance, 16, 766-780.

FoLK, M. D., \& LUCE, R. D. (1987). Effects of stimulus complexity on mental rotation rate of polygons. Journal of Experimental Psychology: Human Perception \& Performance, 13, 395-404.

Fortin, C., \& Rousseau, R. (1987). Time estimation as an index of processing demand in memory search. Perception \& Psychophysics, 42, 377-382.

Fortin, C., Rousseau, R., Bourque, P., \& Kirouac, E. (1993). Time estimation and concurrent nontemporal processing: Specific interference from short-term-memory demands. Perception \& Psychophysics, 53, 536-548.

GetTY, D. J. (1976). Counting processes in human timing. Perception \& Psychophysics, 20, 191-197.

Gibbon, J., ChuRCH, R. M., \& Meck, W. H. (1984). Scalar timing in memory. In J. Gibbon \& L. Allan (Eds.), Timing and time perception (Annals of the New York Academy of Sciences, Vol. 423, pp. 52-77). New York: New York Academy of Sciences.

Hicks, R. E., Miller, G. W., Gaes, G., \& Bierman, K. (1977). Concurrent processing demands and the experience of time-in-passing. American Journal of Psychology, 90, 431-446.

Hicks, R. E., Miller, G. W., \& Kinsbourne, M. (1976). Prospective and retrospective judgments of time as a function of amount of information processed. American Journal of Psychology, 89, 719730 .

Kantowitz, B. H., \& KNIGHT, J. L. (1978). When is an easy task difficult and vice versa? A reply to Lane. Acta psychologica, 42, 163170.

KIRK, R. E. (1982). Experimental design: Procedures for the behavioral sciences (2nd ed.). Belmont, CA: Brooks/Cole.

LoGAN, G. D. (1979). On the use of a concurrent memory load to measure attention and automaticity. Journal of Experimental Psychology: Human Perception \& Performance, 5, 189-207.

LOGIE, R. H., \& SALWAY, A. S. (1990). Working memory and modes of thinking: A second task approach. In K. J. Gilhooly, M. T. G. Keane, R. H. Logie, \& G. Erdos (Eds.), Lines of thinking (pp. 99-113). New York: Wiley.

Meck, W. H. (1984). Attentional bias between modalities: Effect on the interval clock, memory, and decision stages used in animal time discrimination. In J. Gibbon \& L. Allan (Eds.), Timing and time perception (Annals of the New York Academy of Sciences, Vol. 423, pp. 528-541). New York: New York Academy of Sciences.

METZleR, J., \& SHEPaRd, R. N. (1974). Transformational studies of the internal representation of three-dimensional objects. In R. Solso (Ed.), Theories in cognitive psychology: The Loyola Symposium (pp. 147-201). Potomac, MD: Erlbaum.

Michon, J. A. (1975). Time experience and memory processes. In J. T. Fraser \& N. Lawrence (Eds.), The study of time (Vol. 2, pp. 302313). New York: Springer-Verlag.

Petrusic, W. M. (1984). Explicit counting and time-order errors in du- ration discrimination. In J. Gibbon \& L. Allan (Eds.), Timing and time perception (Annals of the New York Academy of Sciences, Vol. 423, pp. 630-633). New York: New York Academy of Sciences. Rousseau, R., Picard, D., \& Pitre, E. (1984). An adaptive counter model for time estimation. In J. Gibbon \& L. Allan (Eds.), Timing and time perception (Annals of the New York Academy of Sciences, Vol. 423 pp. 639-642). New York: New York Academy of Sciences. SCHNEIDER, W., \& DETWEILER, M. (1987). A connectionist/control architecture for working memory. In G. H. Bower (Ed.), The psychology of learning and motivation (Vol. 21, pp. 54-119). New York: Academic Press.

Schneider, W., \& Shiffrin, R. M. (1977). Controlled and automatic human information processing: I. Detection, search, and attention. Psychological Review, 84, 1-66.

Shepard, D., \& Metzler, J. (1971). Mental rotation of threedimensional objects. Science, 171, 701-703.

Shepard, S., \& Metzler, D. (1988). Mental rotation: Effects of dimensionality of objects and type of task. Journal of Experimental Psychology: Human Perception \& Performance, 14, 3-11.

SiDOWSKI, J. B. (ED.) (1966). Experimental methods and instrumentation in psychology. New York: McGraw-Hill.

Treisman, A., \& Gelade, G. (1980). A feature integration theory of attention. Cognitive Psychology, 12, 97-136.

VANDERPLAS, J. M., \& GARVIN, E. A. (1959). The association value of random shapes. Journal of Experimental Psychology, 57, 147-154.

Wickens, C. D. (1984). Processing resources in attention. In R. Parasuraman \& D. T. Davies (Eds.), Varieties of attention (pp. 63-102). New York: Academic Press.

WiLDING, J., \& WhITE, W. (1985). Impairment of rhyme judgements by silent and overt articulatory suppression. Quarterly Journal of Experimental Psychology, 37A, 95-107.

ZAKAY, D. (1993). Relative and absolute duration judgments under prospective and retrospective paradigms. Perception \& Psychophysics, 54, 656-664.

\section{APPENDIX Examples of Stimuli Used in Experiment 2}

Rhyming

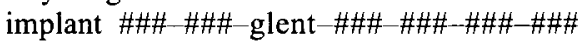
assez \#\#\#-\#\#\#-\#\#\#-drer-\#\#\#-\#\#\#-\#\#\#

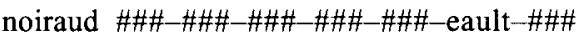
jaloux \#\#\# \#\#\#-gond-\#\#--blout \#\#\# \#\#\# amer \#\#\#-\#\#\#-\#\#\#-\#\#\#-von-plair-\#\#\# radeau \#\#\#-kon-flo-tant-\#\#\#-beur-\#\#\# noiraud \#\#\#-deau-cal-\#\#\#-ceu-vi-\#\#\#

Nonrhyming

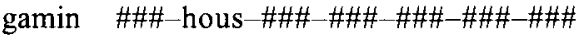
implant \#\#\#-\#\#\#-plau-\#\#\#-\#\#\#-\#\#\#-\#\#\#

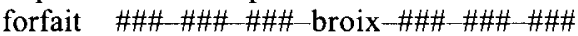
ennui \#\#\#-\#\#\#-\#\#\#-\#\#\#-blair-\#\#\#-\#\#\# rapport \#\#\#-\#\#\#-\#\#\#-\#\#\#-\#\#\#-dair-\#\#\# carnet \#\#\# \#\#\# \#\#\# \#\#\#-con-vat-\#\#\# radeau \#\#\#-gan-fort-\#\#\#-\#\#\#-\#\#\#-\#\#\#

(Manuscript received June 15, 1993; revision accepted for publication August 10, 1994.) 